\title{
Testagem universal de COVID-19 na população obstétrica: impactos para a saúde pública
}

\author{
Universal COVID-19 testing in the obstetric \\ population: impacts on public health
}

\author{
Detección universal de COVID-19 en la población \\ obstétrica: impactos en la salud pública
}

\author{
Mariane de Oliveira Menezes 1 \\ Carla Betina Andreucci 2 \\ Marcos Nakamura-Pereira 3 \\ Roxana Knobel 4 \\ Cláudia Garcia Magalhães 1 \\ Maíra Libertad Soligo Takemoto 1
}

doi: 10.1590/0102-311X00164820

Em 11 de março de 2020, a Organização Mundial da Saúde (OMS) declarou a COVID-19 como pandemia mundial. Como o surgimento da condição é recente, várias questões permanecem por ser esclarecidas. Por exemplo, ainda restam dúvidas a respeito do potencial de transmissão por portadores assintomáticos e pré-sintomáticos, não há, até o momento, tratamento específico comprovadamente eficaz, como também não se sabe se há ou quanto dura a imunidade adquirida após o contágio. Medidas com vistas à redução da transmissão são preconizadas, como isolamento social, higiene e uso de máscaras faciais, além de equipamentos de proteção individual específicos para profissionais de saúde 1,2,3.

Até 7 de julho de 2020, a doença já havia causado 542.798 óbitos no mundo 4 e chama a atenção a diversidade de padrões clínicos e multiplicidade de órgãos e sistemas afetados. No entanto, seu efeito durante o ciclo gravídico-puerperal não é ainda totalmente conhecido. Dados iniciais pareciam mostrar que não havia maior susceptibilidade de gestantes e puérperas à COVID-19 5,6. No entanto, dados mais recentes vêm indicando a possibilidade de desfechos desfavoráveis, talvez relacionada às adaptações do organismo à gravidez, em especial sobre os sistemas cardiovascular e imunológico, também afetados pelos coronavírus 7,8,9,10,11. Embora evidências da transmissão vertical da doença ainda sejam escassas, há relatos de infecção neonatal 12, além de aumento do risco de prematuridade em decorrência do agravamento do quadro clínico de gestantes com a COVID-19 6,10, ampliando os potenciais impactos da doença na gestação para além dos efeitos imediatos na saúde materna ou fetal. Adicionalmente, preocupações relacionadas ao aumento do risco de desfechos maternos e perinatais desfavoráveis em contexto de poucos recursos vêm sendo levantadas na literatura, em particular para o Brasil e outros países da América Latina 7,13. Atualmente, mortes maternas associadas à COVID-19 publicadas no mundo somam, até 7 de julho de 2020, 160 casos, sendo eles 7 no Irã, 7 no México, 5 no Reino Unido, 16 nos Estados Unidos, 1 na França e 124 no Brasil 9,10,14,15,16,17.

Um aspecto da COVID-19 bastante relevante para a saúde pública é o desconhecimento da prevalência do vírus na população geral assintomática ou oligossintomática com quadros virais inespecíficos. Em todo o mundo, há reconhecida dificuldade de testagem universal da população, especialmente em países de baixa e média rendas 18. Mesmo antes da pandemia, o Brasil vinha encontrando dificuldades em reduzir e até mesmo manter as taxas de mortalidade materna ${ }^{19}$. Nesse contexto, a determinação da prevalência de COVID-19 em gestantes, parturientes e puérperas é fundamental para o planejamento estratégico do cuidado obstétrico e neonatal.
1 Faculdade de Medicina de Botucatu, Universidade Estadual Paulista, Botucatu, Brasil. 2 Centro de Ciências Biológicas e da Saúde, Universidade Federal de São Carlos, São Carlos, Brasil.

3 Instituto Nacional de Saúde da Mulher, da Criança e do Adolescente Fernandes Figueira, Fundação Oswaldo Cruz, Rio de Janeiro, Brasil. 4 Departamento de Ginecologia e Obstetrícia, Universidade Federal de Santa Catarina, Florianópolis, Brasil.

\section{Correspondência} M. O. Menezes Faculdade de Medicina de Botucatu, Universidade Estadual Paulista. Av. Prof. Mário Rubens Guimarães Montenegro $s / n$, Botucatu, SP 18618-687, Brasil.

mariane.menezes@unesp.br 
Em razão da sobrecarga universal do sistema de saúde (crônica e agravada com as demandas da pandemia), barreiras de acesso têm dificultado o seguimento pré-natal de gestantes de risco habitual e de alto risco, o que tem sido descrito internacionalmente como um fator desencadeador de piores desfechos maternos e neonatais 20 . A testagem universal da população obstétrica poderia subsidiar o planejamento da assistência ao parto durante a pandemia 21,22. Aspectos que seriam diretamente impactados pelo conhecimento do diagnóstico de COVID-19 em gestantes e puérperas assintomáticas, pré-sintomáticas ou oligossintomáticas incluem:

(i) Avaliação da necessidade de reestruturação organizacional das enfermarias de alojamento conjunto - onde internam-se puérperas e seus bebês, frequentemente em ambiente compartilhado, muitas vezes exíguo ${ }^{3}$;

(ii) Adequação do uso, fornecimento e distribuição de equipamento de proteção individual (EPIs) de modo racional, com vistas à proteção da equipe de saúde, população também muito vulnerável à infecção pelo coronavírus 23 ;

(iii) Adoção oportuna de medidas de prevenção de infecção no neonato, no momento do parto, pós-parto imediato e alojamento conjunto, incluindo orientações específicas para a manutenção da amamentação 24;

(iv) Orientações adequadas para alta hospitalar, incluindo educação em saúde para a manutenção do isolamento domiciliar e precauções para reduzir a transmissão no domicílio.

Seis publicações de séries de casos avaliaram programas de testagem universal para COVID-19 em gestantes admitidas em maternidades, em trabalho de parto, por outra razão obstétrica ou intercorrências clínicas (Tabela 1). Os estudos foram conduzidos nos Estados Unidos, Reino Unido, Portugal e Japão 21,22,25,26,27,28. De modo geral, a conclusão desses estudos aponta a testagem universal como uma estratégia que impactaria positivamente o planejamento de ações de gestão e cuidado, tanto do ponto de vista clínico (melhor monitoramento das gestantes e puérperas com diagnóstico confirmado de COVID-19) quanto organizacional (adoção de medidas de prevenção de transmissão para profissionais, população obstétrica em geral e bebês) e científico (reconhecimento da prevalência da COVID-19 neste subgrupo).

Nesses estudos, as mulheres foram testadas independentemente da presença de sintomas de COVID-19 ou contato com casos conhecidos da doença por meio da coleta de swabs na admissão, analisados usando-se a técnica de RT-PCR (reverse-transcriptase polymerase chain reaction). Conforme a Tabela 1, os resultados das publicações de Nova Iorque, Estados Unidos, apresentaram maior porcentagem de casos positivos entre os estudos citados. Esse achado é esperado, uma vez que, no momento das testagens, Nova Iorque tinha a pior situação epidemiológica (Figura 1). O percentual de gestantes positivas para COVID-19 variou de 3,8-11,7\% nos demais contextos.

Em face de diferenças nos critérios para testagem, definição de casos e medidas de contenção em cada país e contexto local, a habilidade de comparar esses dados ou de projetar sua aplicabilidade para outros contextos é dificultada. No entanto, utilizando as informações de casos cumulativos de COVID-19 em cada contexto na data final de coleta de dados dos referidos estudos 29,30,31 e dados de população 32,33, é possível calcular a proporção de casos por COVID-19 por milhão de habitantes em cada contexto (Figura 1). Com base nessa contextualização, é possível antecipar que, no Brasil, por

Tabela 1

Achados das séries de casos sobre rastreamento universal de COVID-19 em população obstétrica.

\begin{tabular}{|c|c|c|c|c|}
\hline Estudo & País & $\begin{array}{l}\text { Tamanho da } \\
\text { amostra }\end{array}$ & $\begin{array}{c}\text { Mulheres com teste positivo } \\
\text { para SARS-CoV-2 (\%) }\end{array}$ & $\begin{array}{c}\text { Mulheres } \\
\text { assintomáticas (\%) }\end{array}$ \\
\hline Campbell et al. 27 & Estados Unidos (New Haven, Connecticut) & 770 & 3,9 & 73,3 \\
\hline Sutton et al. 21 & Estados Unidos (Nova Iorque) & 214 & 15,4 & 87,9 \\
\hline Vintzileos et al. 28 & Estados Unidos (Nova Iorque) & 161 & 19,9 & 66,6 \\
\hline Khalil et al. 22 & Reino Unido (Londres) & 129 & 7,0 & 88,9 \\
\hline Doria et al. 25 & Portugal (Norte) & 103 & 11,7 & 91,6 \\
\hline Ochiai et al. 26 & Japão (Tóquio) & 52 & 3,8 & 100,0 \\
\hline
\end{tabular}


Figura 1

Prevalência de COVID-19 em gestantes e proporção de casos de COVID-19 por milhão de habitantes em cada contexto.

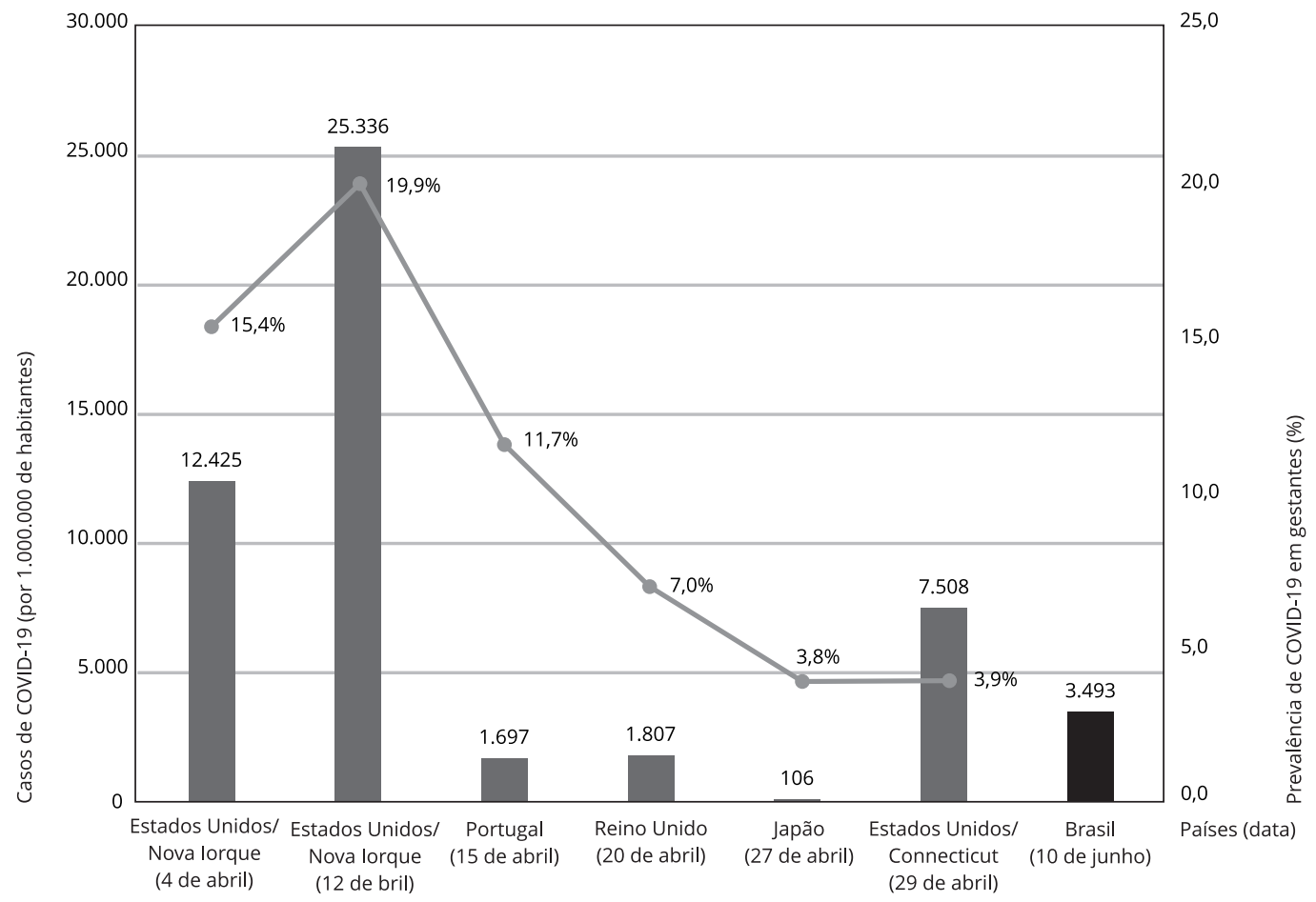

comparação com os demais locais, teríamos um cenário pelo menos intermediário de prevalência de COVID-19 em gestantes, caso políticas de testagem universal fossem adotadas. Se considerarmos que diferenças nos números de testes realizados por milhão de habitantes entre os países analisados refletem magnitudes diferentes de subnotificação, talvez fosse razoável antecipar um quadro até mais crítico no Brasil. Por exemplo, nos três cenários dos Estados Unidos teríamos número de testes por milhão de habitantes entre 15.538 e 26.594 29,30,33. No Brasil, em 10 de junho, os testes registrados para COVID-19 representavam 4.706/milhão de habitantes ${ }^{4}$, indicando potencialmente que exista um número bastante mais alto de casos do que os oficialmente documentados, o que consequentemente refletiria na prevalência de COVID-19 na população obstétrica.

Esses estudos trazem ainda dados a respeito do porcentual de gestantes com COVID-19 que se apresentavam assintomáticas na admissão, que variou de 66,6\% a 100\% (Tabela 1). Convém destacar que a definição de caso suspeito em muitos contextos no Brasil ainda inclui necessariamente a presença de febre, o que limita a elegibilidade para a testagem mesmo de casos internados com outros sintomas sugestivos de COVID-19, mas que não apresentam febre. Uma revisão da literatura compilando dados de séries de casos observou, de forma similar, que apenas cerca de $50 \%$ das pacientes obstétricas com COVID-19 apresentavam febre na admissão 12. Conforme Boletim Epidemiológico 34 do Ministério da Saúde, que reporta o perfil de gestantes com síndrome respiratória aguda grave (SRAG) por COVID-19 no país, 72,9\% dos casos tinham febre, o que demonstra que, mesmo entre os casos graves, um percentual relevante não apresenta febre.

Esses dados reforçam a necessidade da testagem universal de pacientes obstétricas como uma estratégia urgente para a proteção de mulheres gestantes ou puérperas e seus bebês, e também de profissionais de saúde durante a vigência da pandemia, possibilitando o planejamento adequado dos fluxos de encaminhamento, da atenção ao parto e aumento da vigilância direcionada à prevenção de 
óbitos e near miss. A testagem universal ajudará a diminuir o impacto da pandemia sobre as mulheres, principalmente as gestantes de maior vulnerabilidade, sobre as quais recai a maior carga da mortalidade materna.

\section{Colaboradores}

M. O. Menezes e M. L. S. Takemoto participou da concepção e planejamento do estudo, bem como da coleta e análise de dados, redação da versão inicial do manuscrito e revisão e aprovação da versão final. C. B. Andreucci, M. Nakamura-Pereira, R. Knobel e C. G. Magalhães participaram da concepção e planejamento do estudo, análise de dados, redação da versão inicial do manuscrito e revisão e aprovação da versão final.

\section{Referências}

1. Agência Nacional de Vigilância Sanitária. Nota Técnica GVIMS/GGTES/ANVISA, n. 04/2020. Orientações para serviços de saúde: medidas de prevenção e controle que devem ser adotadas durante a assistência aos casos suspeitos ou confirmados de infecção pelo novo coronavírus (SARS-CoV-2). https://www20.anvisa.gov.br/ segurancadopaciente/index.php/noticias/176nota-tecnica-n-04-2020-gvims-ggtes-anvisa -atualizada (acessado em 10/Jun/2020).

2. Departamento de Atenção Hospitalar, Domiciliar e de Urgência, Secretaria de Atenção Especializada, Ministério da Saúde. Protocolo de Manejo Clínico da Covid-19 na Atenção Especializada. Brasília: Ministério da Saúde; 2020.

3. Secretaria de Atenção Primária à Saúde, Ministério da Saúde. Nota Técnica COSMU/CGCIVI/ DAPES/SAPS/MS no 12/2020. https://portal deboaspraticas.iff.fiocruz.br/biblioteca/notatecnica-no-12-2020-cosmu-cgcivi-dapes-sapsms/ (acessado em 10/Jun/2020).

4. Worldometer. Coronavirus cases. https://www. worldometers.info/coronavirus/coronaviruscases/\#daily-cases (acessado em 10/Jun/2010).

5. Chen L, Li Q, Zheng D, Jiang H, Wei Y, Zou L, et al. Clinical characteristics of pregnant women with Covid-19 in Wuhan, China. N Engl J Med 2020; 382:e100.

6. Yan J, Guo J, Fan C, Juan J, Yu X, Li J, et al. Coronavirus disease 2019 (COVID-19) in pregnant women: a report based on 116 cases. Am J Obstet Gynecol 2020; 223:111.e1-111.e14.

7. Amorim MMR, Takemoto MLS, Fonseca EB. Maternal deaths with coronavirus disease 2019: a different outcome from low- to middleresource countries? Am J Obstet Gynecol 2020; [Online ahead of print].

\section{Informações adicionais}

ORCID: Mariane de Oliveira Menezes (0000-00028525-0521); Carla Betina Andreucci (0000-00025590-108X); Marcos Nakamura-Pereira (00000002-4231-0205); Roxana Knobel (0000-00019180-4685); Cláudia Garcia Magalhães (00000001-7033-1807); Maíra Libertad Soligo Takemoto (0000-0002-7016-2879).

8. Breslin N, Baptiste C, Gyamfi-Bannerman C, Miller R, Martinez R, Bernstein K, et al. COVID-19 infection among asymptomatic and symptomatic pregnant women: two weeks of confirmed presentations to an affiliated pair of New York City hospitals. Am J Obstet Gynecol MFM 2020; 2:100118.

9. Hantoushzadeh S, Shamshirsaz AA, Aleyasin A, Seferovic MD, Aski SK, Arian SE, et al. Maternal death due to COVID-19 disease. Am J Obstet Gynecol 2020; 223:109.E1-109.E16.

10. Knight M, Bunch K, Vousden N, Morris E, Simpson N, Gale C, et al. Characteristics and outcomes of pregnant women admitted to hospital with confirmed SARS-CoV-2 infection in UK: national population based cohort study BMJ 2020; 369:m2107.

11. Collin J, Byström E, Carnahan A, Ahrne M. Pregnant and postpartum women with SARSCoV-2 infection in intensive care in Sweden. Acta Obstet Gynecol Scand 2020; 99:819-22.

12. Juan J, Gil MM, Rong Z, Zhang Y, Yang H, Poon LCY. Effects of coronavirus disease 2019 (COVID-19) on maternal, perinatal and neonatal outcomes: a systematic review of 266 pregnancies. Ultrasound Obstet Gynecol 2020; 56:15-27.

13. Buekens P, Alger J, Bréart G, Cafferata ML, Harville E, Tomasso G. A call for action for COVID-19 surveillance and research during pregnancy. Lancet Glob Health 2020; 8:e877-8.

14. Kayem G, Lecarpentier E, Deruelle P, Bretelle F, Azria E, Blanc J, et al. A snapshot of the Covid-19 pandemic among pregnant women in France. J Gynecol Obstet Hum Reprod 2020; 101826. 
15. Lumbreras-Marquez MI, Campos-Zamora M, Lizaola-Diaz de Leon H, Farber MK. Maternal mortality from COVID-19 in Mexico. Int J Gynaecol Obstet 2020; 150:266-7.

16. Ellington S, Strid P, Tong VT, Woodworth K, Galang RR, Zambrano LD, et al. Characteristics of women of reproductive age with laboratory-confirmed SARS-CoV-2 infection by pregnancy status - United States, January 22-June 7, 2020. MMWR Morb Mortal Wkly Rep 2020; 69:769-75.

17. Takemoto MLS, Menezes MO, Andreucci CB, Nakamura-Pereira M, Amorim MMR, Katz L, et al. The tragedy of COVID-19 in Brazil: 124 maternal deaths and counting. Int J Gynaecol Obstet 2020; [Online ahead of print].

18. Hopman J, Allegranzi B, Mehtar S. Managing COVID-19 in low- and middle-income countries. JAMA 2020; 323:1549-50.

19. Cirelli JF, Surita FG, Costa ML, Parpinelli MA, Haddad SM, Cecatti JG. The burden of indirect causes of maternal morbidity andmortality in the process of obstetric transition: a crosssectional multicenter study. Rev Bras Ginecol Obstet 2018; 40:106-14.

20. Roberton T, Carter ED, Chou VB, Stegmuller AR, Jackson BD, Tam Y, et al. Early estimates of the indirect effects of the COVID-19 pandemic on maternal and child mortality in low-income and middle-income countries: a modelling study. Lancet Glob Health 2020; 8:E901-8.

21. Sutton D, Fuchs K, D'Alton M, Goffman D. Universal screening for SARS-CoV-2 in women admitted for delivery. N Engl J Med 2020; 382:2163-4.

22. Khalil A, Hill R, Ladhani S, Pattisson K, O’Brien P. SARS-CoV-2 in pregnancy: symptomatic pregnant women are only the tip of the iceberg. Am J Obstet Gynecol 2020; [Epub ahead of print].

23. Bandyopadhyay S, Baticulon RE, Kadhum M, Alser M, Ojuka DK, Badereddin Y, et al. Infection and mortality of healthcare workers worldwide from COVID-19: a scoping review. medRxiv 2020; 2 jun. https://www.medrxiv. org/content/10.1101/2020.06.04.20119594v1.

24. Secretaria de Atenção Primária à Saúde, Ministério da Saúde. Nota Técnica DAPES/SAPS/ MS no 7/2020. https://portaldeboaspraticas. iff.fiocruz.br/biblioteca/gestantes-nota-tecni ca-no-6-2020-cosmu-cgcivi-dapes-saps-ms/ (acessado em 10/Jun/2020).
25. Dória M, Peixinho C, Laranjo M, Varejão AM, Silva PT. Covid-19 during pregnancy: a case series from an universally tested population from the north of Portugal. Eur J Obstet Gynecol Reprod Biol 2020; 250:261-2.

26. Ochiai D, Kasuga Y, Iida M, Ikenoue S, Tanaka M. Universal screening for SARS-CoV-2 in asymptomatic obstetric patients in Tokyo, Japan. Int J Gynecol Obstet 2020; 150:268-9.

27. Campbell KH, Tornatore JM, Lawrence KE, Illuzzi JL, Sussman LS, Lipkind HS, et al. Prevalence of SARS-CoV-2 among patients admitted for childbirth in Southern Connecticut. JAMA 2020; 323:2520-2.

28. Vintzileos W, Muscat J, Hoffmann E, Vo D, John $\mathrm{N}$, Vertichio R, et al. Screening all pregnant women admitted to labor and delivery for the virus responsible for COVID-19. Am J Obstet Gynecol 2020; [Epub ahead of print].

29. Department of Health, New York State. New York State statewide COVID-19 testing. https://health.data.ny.gov/Health/New-YorkState-Statewide-COVID-19-Testing/xdss-u53e (acessado em 10/Jun/2020).

30. Connecticut Open Data. Office of policy and management. COVID-19 tests, cases, hospitalizations and deaths (Statewide). 2020. https:// data.ct.gov/Health-and-Human-Services/ COVID-19-Tests-Cases-Hospitalizations-andDeaths-S/rf3k-f8fg (acessado em 10/Jun/2020).

31. World Health Organization. COVID-19 situation reports. 2020. https://www.who.int/emer gencies/diseases/novel-coronavirus-2019/situation-reports (acessado em 8/Jun/2020).

32. The World Bank. The World Bank data: population, total. 2020. https://data.worldbank.org/ indicator/sp.pop.totl (acessado em 10/Jun/ 2020).

33. U.S. Census Bureau. QuickFacts: United States. 2020. https://www.census.gov/quickfacts/ fact/table/US/PST045219 (acessado em 10/ Jun/2020).

34. Secretaria de Vigilância em Saúde, Ministério da Saúde. Boletim Epidemiológico Especial: COE-COVID 19 2020; (17). https://www.sau de.gov.br/images/pdf/2020/May/29/2020-0525---BEE17---Boletim-do-COE.pdf.
Recebido em 12/Jun/2020

Versão final reapresentada em 09/Jul/2020

Aprovado em 11/Jul/2020 